\title{
Numerical Simulation of Wind Drift of Arrows on the Olympic Venue for Tokyo 2020
}

\author{
By Julio Ortiz ${ }^{*}$, Masato Ando ${ }^{ \pm}$\& Takeshi Miyazaki ${ }^{*}$
}

\begin{abstract}
In the present work, the dynamics of archery arrows are studied by means of a mathematical model to evaluate their response to different wind characteristics and initial conditions. Numerical computations are performed to quantify the effect that the background wind has on the dynamics of the arrows during their flights. Aerodynamic and physical characteristics of two commercial arrows are considered. The background wind information from the venue, where the Archery competition will be held in the Tokyo Olympic Games in 2020 was provided by JAMSTEC. In a simulated archery range of $70 \mathrm{~m}$, the heavier arrow showed a lateral deviation from the center of the target of $\sim 0.11 \mathrm{~m}$, whereas the lighter arrow showed a final deviation in its trajectory of $\sim 0.15 \mathrm{~m}$. The ratio of the drag force to the gravitational force plays a key role in determining the deviation in the trajectory. By keeping the boundary layer laminar, a less deviated shot can be achieved. With increasing arrow's initial velocity, the deviation in the trajectory also reduces.
\end{abstract}

Keywords: Background wind, Numerical computation, Olympic Games, Tokyo 2020, Trajectory and attitude of archery arrows.

\section{Introduction}

In this paper, the effect of different types of background winds in the dynamics of archery arrows is studied by means of numerical simulations. Archery competition is a sports discipline in which the accuracy and precision are key factors in order to obtain a good final score. In the competitions using a recurve bow, the archers aim at a target with $1.22 \mathrm{~m}$ in diameter and located $70 \mathrm{~m}$ away. The target is divided into 10 smaller evenly spaced concentric circles, rings. The innermost of the rings has a diameter of $0.122 \mathrm{~m}$ and is assigned with the maximum of 10 points. The archers shoot a specified number of arrows and the one who sums more points wins the competition. Striking the innermost ring gives the opportunity to elevate the final score, which is complicated if the multiple elements affecting the shots are considered.

In recent years, the sports engineering became a flourishing research field for engineers due to the realization of the positive impact of using modern tools and techniques in the design and testing stages of sports equipment. The literature

\footnotetext{
*PhD Student, Graduate School of Informatics and Engineering, University of Electro-Communications, Japan.

${ }^{ \pm}$MSc Student, Graduate School of Informatics and Engineering, University of Electro-Communications, Japan.

Trofessor, Graduate School of Informatics and Engineering, University of Electro-Communications, Japan.
} 
covers a wide range of examples for various sports and approaches (Allen and Goff 2018, Haake 2009), including sport projectiles (Goff 2013, Hubbard 1984).

The archery competition has been extensively studied. On the one hand, the internal ballistics of the arrows, i.e. when the arrow is propelled using the bow, has been analysed by Kooi and Sparenberg (1997), Kooi (1998) and Zaniewsky (2009). Kooi described in detail the flexural oscillation of the arrows during the release stage by assuming the arrows to be inextensible Euler-Bernoulli beams, whereas Zaniewsky analysed the vibrations in the bow-arrow system (Kooi and Sparenberg 1997, Kooi 1998, Zaniewsky 2009).

On the other hand, the external ballistics of an archery arrow refer to the stage immediately after the propulsion phase in which an arrow is subject to aerodynamic and gravitational forces. In a $70 \mathrm{~m}$ archery range, arrows shot with an initial velocity of around $57 \mathrm{~ms}^{-1}$ remain in free-flight less than $1.5 \mathrm{~s}$. Arrows traveling downrange rotate and oscillate longitudinally around their center of mass. Such complex movements make difficult the analysis of their dynamics.

Several research groups have analysed the arrow's external ballistics. Barton et al. $(2011,2012)$ designed and mounted an electronic velocity sensing device to measure the launching and impact velocities of hunting arrows shot with a crossbow (Barton et al. 2011, 2012). Park et al. (2011) carried out water channel experiments and UV dye tests to understand the effect that the point's shape has on the arrow's boundary layer (Park et al. 2011). The water channel tests were performed with a maximum Reynolds number $\mathrm{Re}=3.43 \times 10^{4}$ (using the arrow's diameter as the characteristic length) and varying the angle of attack from $0^{\circ}$ to $3^{\circ}$. The transition point from a laminar to a turbulent boundary layer was verified to be sensitive to the point's geometry, Re and angle of attack.

Park (2011) carried out numerical computations to obtain the wind drift of several types of arrows when uniform side-winds blow (Park 2011). In a previous work of our group (Ortiz et al. 2019), the deviations in the trajectories of arrows subject to background wind with laminar and turbulent boundary layers were quantified. For a uniform side wind of $3 \mathrm{~ms}^{-1}$, arrows with laminar boundary layers showed a reduction in the lateral drift by around $0.15 \mathrm{~m}$, which is not negligible, compared with their turbulent counterpart. Furthermore, arrows with larger mass showed less deviated trajectories than the lighter ones.

It is relevant to note that during outdoor archery competitions, the background wind is an important factor that cannot be controlled by the archers and that must be taken into account when studying the dynamics of archery arrows. It is known that the wind currents might be one of the most important elements disturbing the shots (Park 2019). The wind influence on sports competitions has already been studied for other sports disciplines, like the ski jumping (Jung et al. 2018) and javelin throw (Hubbard and Rust 1984). Nevertheless, the existing studies related with archery competition only cover uniform-type wind velocities, which under real meteorological conditions might be unusual.

During actual outdoor competitions, it is likely that the winds' velocity along the archery range change spatially and temporary. Furthermore, the wind direction might vary abruptly, adding difficulty to the shots and provoking an unexpected arrows' behaviour. Such changing wind velocities have been considered previously 
to compute the trajectory of golf shots (Yaghoobian and Mittal 2018); nevertheless, there is no record of such analysis for the archery competition.

To carry out a more realistic simulation of an outdoors archery competition, the effect of non-uniform wind velocities is considered in the current study. The present work takes into account the wind characteristics where the archery competition will be held in the summer Tokyo Olympic Games in 2020. The wind information was computed using a Large Eddy Simulator (LES), developed by the Japan Agency for Marine-Earth Science and Technology (JAMSTEC). The main characteristic of the wind behaviour information provided by JAMSTEC is that the wind component velocities vary with time and position, allowing to test the response of commercial arrows under a realistic environment.

In this paper, we investigate the influence of the mass, initial velocity and initial angular velocity in the dynamics of the archery arrows. Also, the behaviour of the arrows under uniform and changing winds is compared. Finally, the influence of the delay in the transition of the arrow's boundary layer is studied.

This work is organized as follows; in the section "General Characteristics" are provided the descriptions of the arrows, mathematical model, background wind and initial conditions. In the section "Results" the findings from the numerical computations are shown. In the section "Discussions" are studied the cases for which a delay in the transition of the boundary layer exists. In the section "Conclusions" are made the last comments with a brief summary.

\section{General Characteristics}

\section{Description of the Arrows}

Archery arrows are composed of an elongated shaft, a point located at its front, a set of vanes and a nock at its rear part. In this study, the physical and aerodynamic characteristics of two commercial arrows, obtained in previous experiments of our group (Ortiz et al. 2019), are considered. Both studied arrows are manufactured from an aluminium alloy tube with an external carbon sheet as cover. We refer to them as the type-A and type-B arrows.

The physical characteristics mass $(M)$, radius $(r)$, length $(l)$, moments of inertia around the arrow's axis $\left(I_{3}\right)$ and perpendicular to it $(I)$, are listed in Table 1 . The aerodynamic characteristics are listed in Table 2. The parameters $\alpha$ and $\beta$ are related with the lift and pitching moment, respectively. Also, the spin parameter $\left(S_{\mathrm{P}}\right)$ and the initial velocity of the arrows $\left(V_{0}\right)$ are shown. Two different values of the drag coefficients for the laminar, $C_{D}$ (lam), and turbulent, $C_{D}$ (turb), regimes are taken into account in the simulations. The former is used when the ideal initial angular velocity is achieved (see the section "Initial conditions"). Whereas the latter is used when such ideal initial conditions cannot be achieved. The archery arrows are light and flexible, nevertheless the difference of $\sim 0.005 \mathrm{~kg}$ in mass play an important role in their dynamics.

For comparison, we refer to other sports, where the dynamics of the projectiles play a key role, e.g., the javelin throw. Even though archery and javelin 
throw differ in many aspects, the javelins are subject to the same aerodynamic forces as the archery arrows and serve us to remark some important physical characteristics. Javelins are long spears with a mass of around $0.80 \mathrm{~kg}$ and a fineness ratio $f=80$. The initial launching velocity for javelins is about $30 \mathrm{~ms}^{-1}$. While studying the dynamics of flying javelins, Hubbard (1984) found that these projectiles develop large angles of attack during their flight $\left(\gamma_{\max }>35^{\circ}\right)$ leading to large lift forces necessary to increase the range that would make the thrower to get a high score in the competition (Hubbard 1984). Such values of $\gamma$ also induce a large drag component that leads to less precise shots. In javelin throw, precision is not as relevant as in the archery competition.

Table 1. Physical Characteristics of the Arrows

\begin{tabular}{|l|c|c|c|}
\hline Symbol & Name & Type-A & Type-B \\
\hline$r[\mathrm{~m}]$ & Radius & $2.62 \times 10^{-3}$ & $2.40 \times 10^{-3}$ \\
\hline$l[\mathrm{~m}]$ & Length & 0.625 & 0.625 \\
\hline$M[\mathrm{~kg}]$ & Mass & 0.0142 & 0.0197 \\
\hline$I\left[\mathrm{kgm}^{2}\right]$ & Moment of inertia & $6.98 \times 10^{-4}$ & $8.97 \times 10^{-4}$ \\
\hline$I_{3}\left[\mathrm{kgm}^{2}\right]$ & Moment of inertia & $2.81 \times 10^{-7}$ & $3.23 \times 10^{-7}$ \\
\hline
\end{tabular}

Table 2. Aerodynamic Characteristics of the Arrows

\begin{tabular}{|l|c|c|c|}
\hline Symbol & Name & Type-A & Type-B \\
\hline$\alpha[1 / \mathrm{rad}]$ & Parameter alpha & 40.2 & 45.1 \\
\hline$\beta[1 / \mathrm{rad}]$ & Parameter beta & 16.2 & 21.2 \\
\hline$S_{\mathrm{P}}$ & Spin parameter & 0.029 & 0.034 \\
\hline$C_{\mathrm{D}}(\mathrm{lam})$ & Drag coefficient & 1.50 & 1.63 \\
\hline$C_{\mathrm{D}}(\mathrm{turb})$ & Drag coefficient & 2.69 & 3.23 \\
\hline$V_{0}\left[\mathrm{~ms}^{-1}\right]$ & Initial velocity & 57.3 & 56.7 \\
\hline
\end{tabular}

Source: Ortiz at al. (2019).

One important difference between the aerodynamics of arrows and javelins is the position of their corresponding center of pressure c.p. and center of gravity c.g. In javelins, c.p. is found very close to $c . g$., at around $8 \times 10^{-3} \mathrm{~m}$ [Hubbard, 1984]. Whereas in the case of the arrows, c.g. is located in the front part while c.p. in the tail, having a distance of around $0.40 \mathrm{~m}$ between them. The fact that c.p. and c.g. are very close represents an absence of pitching moment in the javelin's flight. Therefore, no counterbalance effect to the increasing angle of attack takes place. The growing magnitude of $\gamma$ allows generating large values of lift and dragging forces. Contrary, the maximum value of the angle of attack computed by Miyazaki et al. (2017) in arrows in free flight, under still air conditions, is around $\gamma_{\max }=0.4^{\circ}$ (Miyazaki et al. 2017). This small angle of attack is a product of the pitching moment-lift force balance effect. Such small $\gamma$ generates smaller drag force and drift in an arrow than in a javelin, allowing precise shots.

\section{Description of the Mathematical Model}

In this study, the equations of a rigid body simulating an archery arrow are computed. Such model disregards the flexural oscillation of the real arrows in free 
flight, which simplifies the study of these projectiles. We define a fixed coordinate system $x, y$ and $z$ with the corresponding unit vectors $\boldsymbol{i}, \boldsymbol{j}$ and $\boldsymbol{k}$, as shown in Figure 1 . Besides the gravitational force $(g)$, exerted on the vertical direction $(z)$, the movement of the arrow is influenced by the background wind, which is $\boldsymbol{U}=\left(u_{\mathrm{x}}, u_{\mathrm{y}}\right.$, $u_{\mathrm{z}}$ ). In the defined coordinate system, $x$ and $y$ form a horizontal plane or the ground. The velocity of the arrow's center of mass is $V=(V \sin \Theta \cos \Phi, V \sin \Theta$ $\sin \Phi, V \cos \Theta)$. Two unit vectors, $\boldsymbol{t}=\boldsymbol{V}-\boldsymbol{U} /|\boldsymbol{V}-\boldsymbol{U}|$ and $\boldsymbol{n}=(\sin \theta \cos \phi, \sin \theta \sin \phi$, $\cos \theta$ ) are considered. The former along the vector sum of the relative velocity of the arrow's center of mass and the wind, $\boldsymbol{V}-\boldsymbol{U}$, whereas, the latter along the arrow's axis, as shown in Figure 1. Consider $\theta$ and $\Theta$, as the angles formed between $\boldsymbol{n}$ and $\boldsymbol{t}$ with $z$, respectively, whereas $\phi$ and $\Phi$ are measured between the ground projection of $\boldsymbol{n}$ and $\boldsymbol{t}$ with $x$, respectively.

As the arrow translates in free-flight, a misalignment between $\boldsymbol{n}$ and $\boldsymbol{t}$ is expected due to the background wind influence. Such misalignment is characterized as the angle of attack $\gamma=\cos ^{-1}[n \cdot t]$. The computation of the time evolution of $\gamma$ is of interest due to from its value can be inferred the state of the boundary layer (Ortiz et al. 2019).

The aerodynamic loads drag $\left(\boldsymbol{F}_{\mathrm{D}}\right)$ and lift $\left(\boldsymbol{F}_{\mathrm{L}}\right)$ must be considered during the flight simulation. These forces are exerted contrarily and perpendicularly to $\boldsymbol{V}-\boldsymbol{U}$, respectively. The drag and lift forces are, respectively:

$$
\begin{aligned}
& \boldsymbol{F}_{\mathrm{D}}=-\frac{1}{2} C_{\mathrm{D}} \rho \pi r^{2}|\boldsymbol{V}-\boldsymbol{U}|(\boldsymbol{V}-\boldsymbol{U}), \\
& \boldsymbol{F}_{\mathrm{L}}=\frac{1}{2} \alpha \rho \pi r^{2}\left[|\boldsymbol{V}-\boldsymbol{U}|^{2} \boldsymbol{n}-\boldsymbol{n} \cdot(\boldsymbol{V}-\boldsymbol{U})(\boldsymbol{V}-\boldsymbol{U})\right],
\end{aligned}
$$

where $C_{\mathrm{D}}$ is the drag coefficient and $\rho$ is the air's density. The parameter $\alpha$ is related to the lift coefficient with $C_{\mathrm{L}}=\alpha \gamma$ and is determined experimentally [Ortiz et al., 2019]. The arrow's radius is $r$.

The angular momentum is $\boldsymbol{L}=\boldsymbol{I n} \times \mathrm{d} \boldsymbol{n} / \mathrm{d} t+I_{3} \omega_{3} \boldsymbol{n}$, where $I$ and $I_{3}$ represent the moments of inertia around the axis perpendicular to $\boldsymbol{n}$ and around it, respectively. $\omega_{3}$ is the axial angular velocity component. Therefore, the equations of motion are expressed as:

$$
\begin{aligned}
M \frac{d V}{d t} & =-M g \boldsymbol{k}+\boldsymbol{F}_{D}+\boldsymbol{F}_{L}, \\
\frac{\mathrm{d} L}{\mathrm{~d} t} & =I \boldsymbol{n} \times \frac{\mathrm{d}^{2} \boldsymbol{n}}{\mathrm{d} t^{2}}+I_{3}\left(\frac{\mathrm{d} \omega_{3}}{\mathrm{~d} t} \boldsymbol{n}+\omega_{3} \frac{\mathrm{d} \boldsymbol{n}}{\mathrm{d} t}\right) \\
& =\frac{1}{2} \beta \rho \pi r^{2} l|\boldsymbol{V}-\boldsymbol{U}| \boldsymbol{n} \times(\boldsymbol{V}-\boldsymbol{U})+N_{3} \boldsymbol{n},
\end{aligned}
$$

where $M$ is the arrow's mass, $g$ is the gravitational acceleration and $N_{3}$ is the axial component of the torque. The parameter $\beta$ is related with the pitching moment coefficient by $C_{\mathrm{M}}=-\beta \gamma$ and it is determined experimentally (Ortiz et al. 2019). Since we do not have any information about $N_{3}$ and then the time evolution of the angular velocity is unknown, is necessary to approximate its value assuming that 
$S_{\mathrm{P}}=\omega_{3} r /|\boldsymbol{V}-\boldsymbol{U}|$ is constant (Miyazaki et al. 2012, Miyazaki et al. 2017). Here, $S_{\mathrm{P}}$ is the spin parameter, which is obtained from wind tunnel experiments. This assumption is valid for small angles of attack, $|\gamma|<3.0^{\circ}$.

To solve such a system composed by Equations 3-4, it is necessary to compute $\mathrm{d} V / \mathrm{d} t, \mathrm{~d} \Theta / \mathrm{d} t, \mathrm{~d} \Phi / \mathrm{d} t, \mathrm{~d} \omega_{\theta} / \mathrm{d} t$ and $\mathrm{d} \omega_{\phi} / \mathrm{d} t$, where $\mathrm{d} \theta / \mathrm{d} t=\omega_{\theta}$ and $\mathrm{d} \phi / \mathrm{d} t=\omega_{\phi}$. The system is solved by using a standard $4^{\text {th }}$ order Runge-Kutta method with a time step $\Delta t=5 \times 10^{-4} \mathrm{~s}$.

Figure 1. Illustration of an Arrow in Free-flight with the Relevant Variables considered in the Mathematical Model

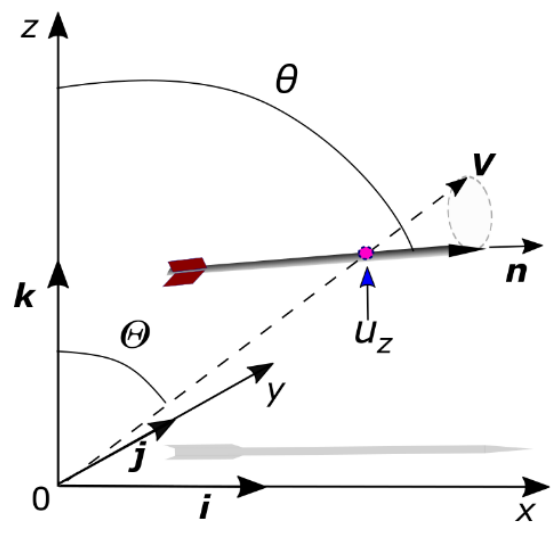

(a)

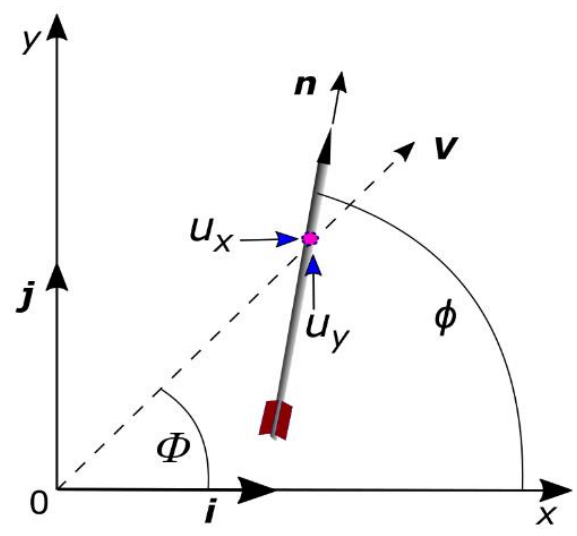

(b)

Source: Authors.

\section{Description of the Simulated Archery Range}

The venue for the archery competitions in the Tokyo Olympic Games 2020 will be held in the semi-closed bay of Tokyo in Yumenoshima Park (Figure 2). The area is located to the east of Tokyo Metropolitan area near to the sea and has a surface of $\sim 95,000 \mathrm{~m}^{2}$. At the moment of writing, the main venue location has been decided but the final design of the archery stadium(s) and facilities are not available for the public yet. Therefore, we project the existence of two possible main competition areas inside the sporting complex. We refer to them as the final round area (fields I, II and III), at the western part of the complex, and the ranking round area (fields IV, V and VI) (Figure 3). Each of the tracks are north oriented with a shooting direction South-North and are simulated to have a dimension of 70 $\mathrm{m} \times 5 \mathrm{~m}$. At $70 \mathrm{~m}$ from the shooting position, it is located a target that has $1.22 \mathrm{~m}$ in diameter. It is recognized that the presence of buildings and the local plant canopy affect seriously the wind patterns (Yaghoobian et al. 2018), which must be taken in consideration in future studies.

Due to the wind effect, the arrows are expected to show deviations in the trajectory, which can be measured at $70 \mathrm{~m}$ using their striking points with the target. Such deviations from the center of the target are $\delta y$ and $\delta z$ in the $y$ and $z$ directions, respectively. The radial deviation from the center of the target is $\delta r=\left(\delta y^{2}+\delta z^{2}\right)^{1 / 2}$. Such deviations are measured and compared for the different types of winds and initial conditions. 
Figure 2. Yumenoshima Park in the Bay of Tokyo, where the Archery Competitions will be held in the Olympic Games in Tokyo 2020

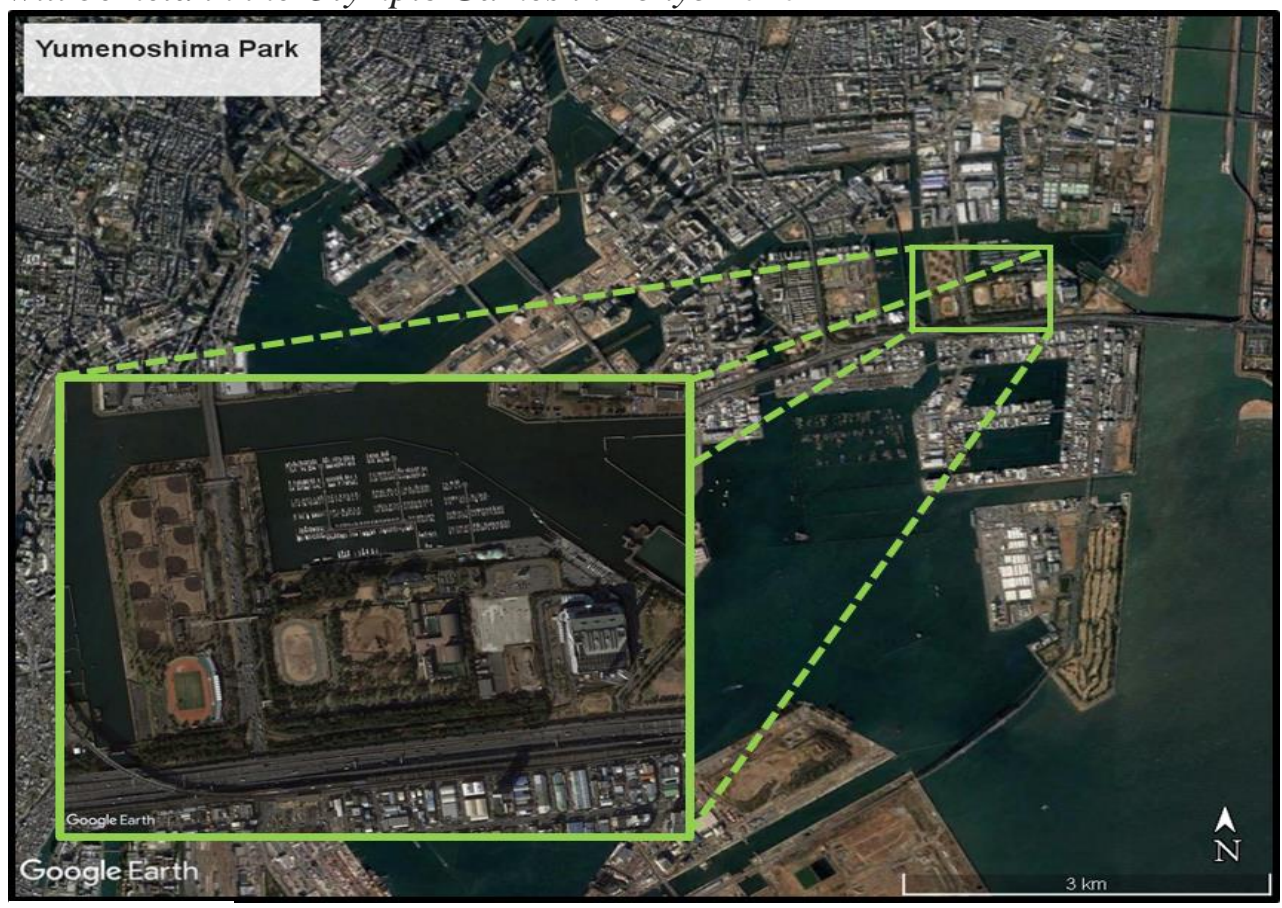

Source: Authors.

Figure 3. Illustration of the Archery Ranges, the Fields I-III are considered as the Final Round Area, whereas the Fields IV-VI are the Ranking Round Area

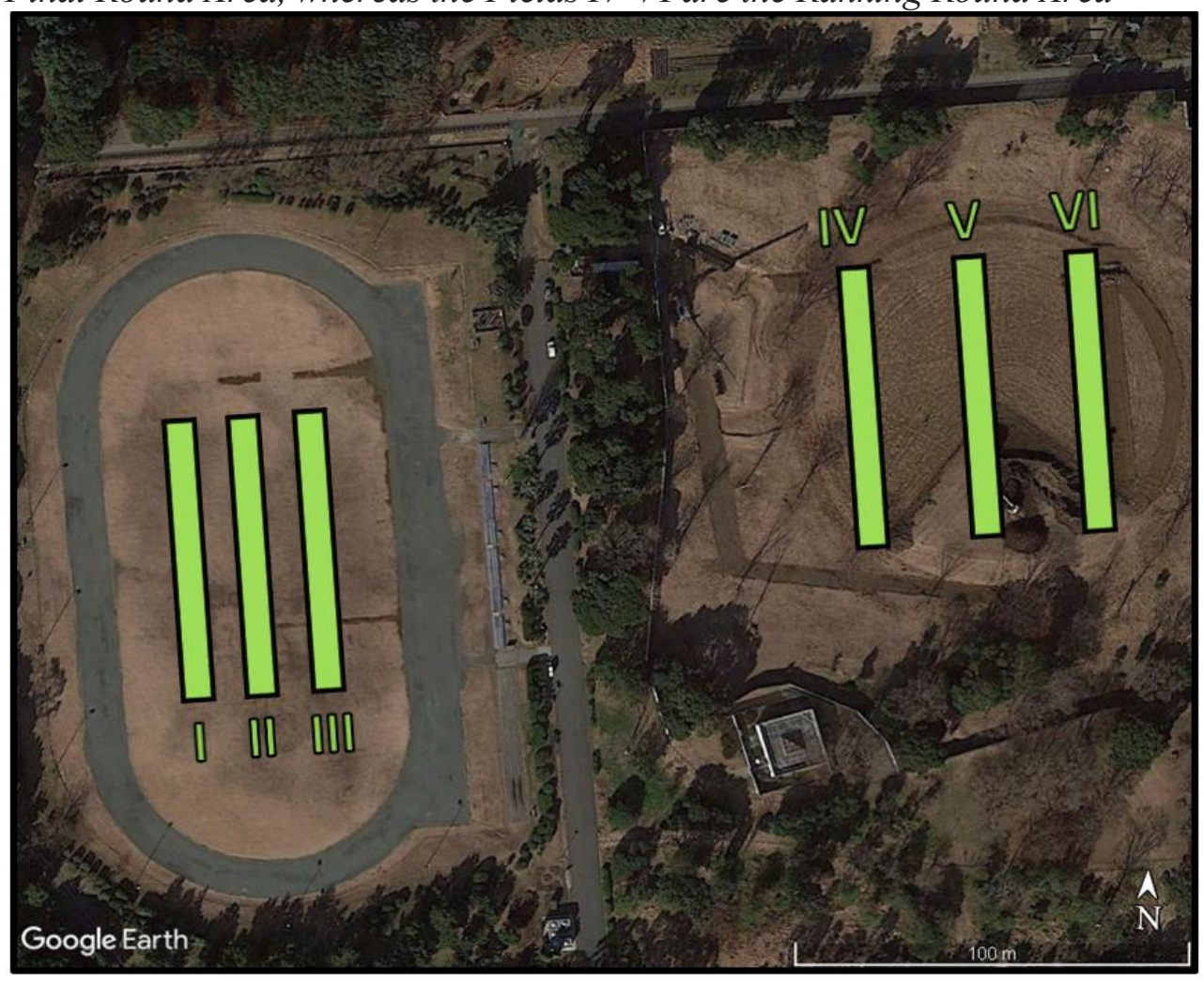

Source: Authors. 
The data corresponding to the time depending wind information in the venue was obtained by means of the high-resolution numerical simulations' results and provided by JAMSTEC. To compute the wind components, they used the Multiscale Simulator for the Geoenvironment (MSSG) model (Matsuda et al. 2018). The MSSG is an atmosphere-ocean coupled general circulation model that, at urban scales, may work as a large-eddy simulator (LES) with the capability to include local building shapes. The simulated wind data describes a typical clear day of August 2007 in the Tokyo bay with sea breeze. This information corresponds to a plane located at $2.5 \mathrm{~m}$ from the ground with a horizontal resolution of $5 \mathrm{~m}$. The wind velocity components change with the position and time during $30 \mathrm{~s}$ with a time step of $0.1 \mathrm{~s}$. In Figures 2 and 3, the original satellite images were obtained from Google Earth Pro and correspond to the current state of the field in October 2018.

\section{Computation of the Background Wind at any Instant and Position}

Because JAMSTEC provides three wind components on a plane at $2.5 \mathrm{~m}$ from the ground level with a horizontal resolution of $5 \mathrm{~m}$, it is necessary to interand extrapolate the wind information at any arrow's position and time. The surface layer approach is used to obtain the wind $\left(u_{x} u_{y}\right.$ and $\left.u_{z}\right)$ that the arrow experiences along its free-flight. The vertically logarithmic wind profile can be described with $u_{x}=u_{x^{*}} / k \ln \left(z / z_{\mathrm{rl}}\right)$ and $u_{y}=u_{y^{*}} / k \ln \left(z / z_{\mathrm{rl}}\right)$. Here, $k$ is the universal von Karman's constant and has an experimentally determined value of $k \approx 0.4$. The value of $z$ is the position of the arrow's center of mass with respect to the ground and $z_{\mathrm{rl}}$ corresponds to the roughness length, whose typical value for a grassy field is $z_{\mathrm{rl}}=0.01 \mathrm{~m}$. The friction velocities $u_{\mathrm{x}^{*}}$ and $u_{\mathrm{y}^{*}}$ are computed for each iteration with:

$$
\begin{aligned}
& u_{x(2.5)}=\frac{u_{x \psi}}{k} \ln \left(\frac{2.5}{z_{\mathrm{rl}}}\right), \\
& u_{y(2.5)}=\frac{u_{y *}}{k} \ln \left(\frac{2.5}{z_{\mathrm{rl}}}\right),
\end{aligned}
$$

where $u_{x(2.5)}$ and $u_{y(2.5)}$ are the $x$ and $y$ components of the wind at $2.5 \mathrm{~m}$ from the ground level and provided by JAMSTEC. Therefore, the wind velocities in the $x$ and $y$ directions at any instant and location are obtained with:

$$
\begin{aligned}
& u_{x}=\frac{u_{x(2.5)} \ln \left(\frac{z}{z_{\mathrm{rl}}}\right)}{\ln \left(\frac{2.5}{z_{\mathrm{rl}}}\right)}, \\
& u_{y}=\frac{u_{y(2.5)} \ln \left(\frac{z}{z_{\mathrm{rl}}}\right)}{\ln \left(\frac{2.5}{z_{\mathrm{rl}}}\right)} .
\end{aligned}
$$


If the condition of continuity is considered, $\nabla \cdot U=0$, the vertical component of the wind, $u_{z}$, can be obtained by using:

$u_{z}=0.0884 u_{z(2.5)}\left[z \ln \left(\frac{z}{z_{\mathrm{rl}}}\right)-z+0.01\right]$,

where $u_{\mathrm{z}(2.5)}$ is the known vertical wind velocity at $2.5 \mathrm{~m}$. The solution of Equations (3-4) allows to know the position, $x_{i}, y_{i}$ and $z_{i}$, of the arrow's center of mass at any instant, $t_{i}$, whereas by solving Equations 7-9, it is possible to obtain the wind components $u_{\mathrm{xi}}, u_{\mathrm{yi}}$ and $u_{\mathrm{zi}}$ for such position. Nevertheless, the change in the arrow's center of mass location is computed in orders of $\mathrm{mm}\left(O\left(10^{-3}\right)\right)$, whereas the spatial resolution of the wind information is $5 \mathrm{~m}$. Therefore, the wind components at any arrow's position were spatially interpolated. Furthermore, the time interval for the wind data provided by JAMSTEC is $0.1 \mathrm{~s}$, whereas the time step in the Runge-Kutta computation is $5 \times 10^{-4} \mathrm{~s}$, resulting necessary to perform a time interpolation of the wind components at each time step. For both the spatial and time interpolations, a cubic spline method was used.

Figure 4 shows the time evolution of the velocity for the three wind components along the total length of the fields II and V at a distance $z=2.5 \mathrm{~m}$ from the ground during $30 \mathrm{~s}$. The wind velocity ranges from $-1 \mathrm{~ms}^{-1}$ to $3.5 \mathrm{~ms}^{-1}$. In Figure $4 \mathrm{a}-\mathrm{b}$, the predominance of South-North (tail-winds, $u_{\mathrm{x}}>0$ ) wind currents is appreciated in both fields due to the presence of the ocean at the south of the archery venue. In the field II at $\sim 30 \mathrm{~m}$, from the shooting position (Figure $4 \mathrm{a}$ ) and $t_{0}=0 \mathrm{~s}$, the tail-winds show a maximum velocity of $u_{\mathrm{x}} \sim 3.2 \mathrm{~ms}^{-1}$ and reduces gradually until $u_{\mathrm{x}} \sim 0.25 \mathrm{~ms}^{-1}$.

Regarding to the side-wind component in the field II (Figure 4c), there exists predominance of positive side-winds $\left(u_{\mathrm{y}}>0\right)$ whereas in the field $\mathrm{V}$ the side-wind velocity remains negative $\left(u_{\mathrm{y}}<0\right)$ during most of the time (Figure $\left.4 \mathrm{c}\right)$. The vertical wind component in Figure $4 \mathrm{e}-\mathrm{f}$ is negative along both tracks, $u_{\mathrm{z}}<0$. As appreciated from the Figure 4, the wind conditions change with the position and the time. Such kind of behaviour might be expected in archery ranges located outdoors. The archer's lack of knowledge of the wind behaviour may lead to an unexpected arrow's trajectory, affecting the final score.

\section{Initial Conditions}

The initial position of the arrow corresponds to the fixed place at which the archer holds it before performing the shot, thus the position of the center of mass is $x_{0}=0, y_{0}=0$ and $z_{0}=1.5 \mathrm{~m}$. The center of the target is located $70 \mathrm{~m}$ away in the positive $x$ direction and $z_{\text {tar }}=1.3 \mathrm{~m}$ from the ground.

We consider $\Phi_{0}=0$ and the value of $\Theta_{0}$ is adjusted so that under still wind conditions, the arrow hits the center of the target. $\Theta_{0}$ depends on the characteristics of the arrow, initial velocity $V_{0}$ and initial angular velocities $\omega_{\theta 0}$ and $\omega_{\phi 0}$., initial velocity $V_{0}$ and initial angular velocities $\omega_{\theta 0}$ and $\omega_{\phi 0}$. The initial velocities for both types of arrows are listed in Table 2. The magnitude of the initial angular velocities plays an important role to reduce the angle of attack and the drag exerted on the 
arrow, as shown by Ortiz et al. (2019). Therefore, two different initial angular velocities are considered in a separated way.

Figure 4. Time Evolution of the Wind Components $u_{x}(\boldsymbol{a}, \boldsymbol{b}), u_{y}(\boldsymbol{c}, \boldsymbol{d})$ and $u_{z}(\boldsymbol{e}, \boldsymbol{f})$ for the Fields II and $V$

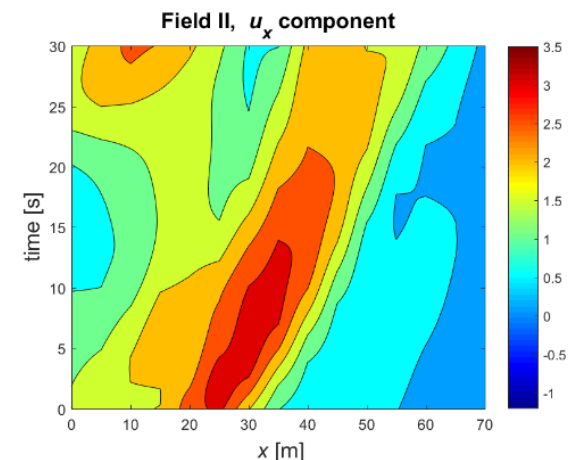

(a)

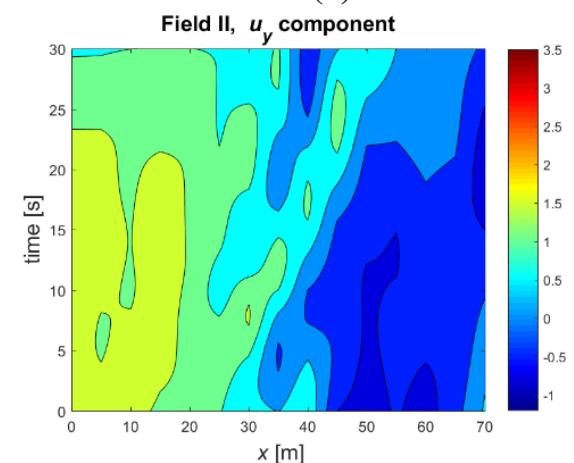

(c)

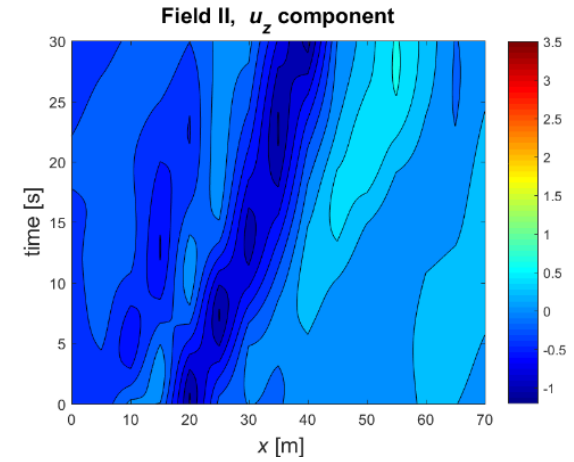

(e)

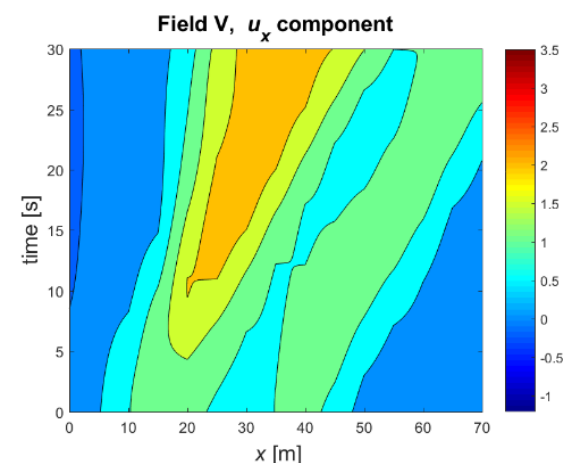

(b)

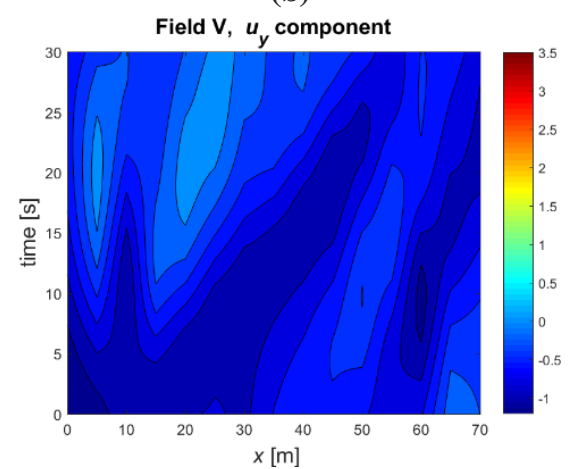

(d)

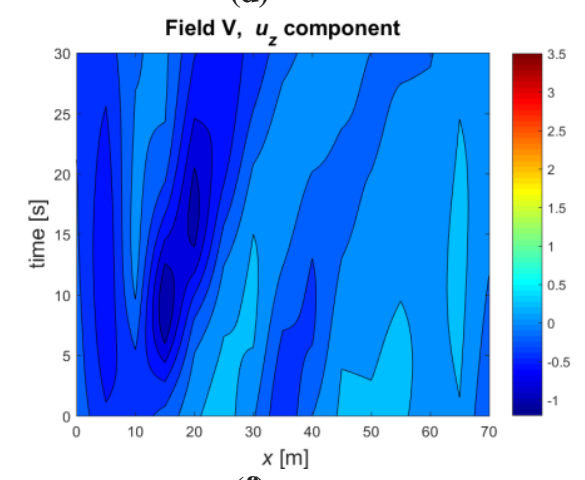

(f)

Source: Authors.

In the former, the angular velocities are assumed to be zero or $(\mathrm{d} n / \mathrm{d} t)_{0}=0$, implying that:

$\omega_{\phi 0}=\omega_{\theta 0}=0$

$\phi_{0}=0$,

$\theta_{0}=\Theta_{0}$. 
The second category is the so-called ideal initial angular velocity, defined as $(\mathrm{d} \boldsymbol{n} / \mathrm{d} t)_{0}=(\mathrm{d} \boldsymbol{t} / \mathrm{d} t)_{0}$. When the ideal initial angular velocity is achieved, the arrow almost aligns with $\boldsymbol{V}$ - $\boldsymbol{U}$ and $\gamma$ remains small enough to preserve the boundary layer laminar (Miyazaki et al. 2017). For these kinds of computations, the laminar value of $C_{\mathrm{D}}$ is used. When the zero initial angular velocity is considered, the turbulent value of $C_{\mathrm{D}}$ is introduced.

\section{Results}

In this section, the results obtained from the numerical computations are presented. In the first subsection the effects of uniform side-, head- and tail-winds in the trajectory are compared. Additionally, the influence of increasing the initial velocity of the arrows under side-winds is described.

Further, the role that the arrows' mass play in their behaviour under velocitychanging wind fields is studied in detail. Finally, the trajectories of arrows for which the boundary layer transition, from laminar to turbulent regimes, takes place during the flight are compared.

\section{Behaviour of the Arrow Subject to a Uniform Background Wind}

As a first approach, it is considered the influence of uniform winds. In these cases, the arrows are subject to the same wind velocity regardless their position. The three studied cases are the side-, head- and tail-winds. The side-wind $\left(u_{\mathrm{y}}\right)$ is exerted along the $y$-axis, whereas in the tail- $\left(u_{\mathrm{x}}>0\right)$ and head-winds $\left(u_{\mathrm{x}}<0\right)$, the air flow is in and against the $x$-direction, respectively. A wind velocity of $3 \mathrm{~ms}^{-1}$ is considered for all the uniform cases.

For the lighter type-A arrow, the time evolution of the angle of attack, $\gamma$, is shown in Figure 5 for the cases with zero initial angular velocity in the main area. The cases with ideal initial angular velocity are represented in the inserted panel in the inferior part of the figure, whereas the panel in the right superior part depicts the target and the points at which the arrows strike on it.

Miyazaki et al. (2017) proposed that the state of the boundary layer might be inferred from the angle of attack, $\gamma$ (Miyazaki et al. 2017). For small angles of attack, $\gamma<0.4^{\circ}$, the boundary layer can be considered laminar, whereas for $\gamma>0.6^{\circ}$ the boundary layer appeared to be turbulent in most of the extension of a type-A arrow. Therefore, the concept of a threshold value of the angle of attack, $\gamma_{\text {thr }}$, can be introduced. If such $\gamma_{\text {thr }}$ value is exceeded, the transition from laminar to turbulent boundary layer would take place.

For the side-wind with zero initial angular velocity (-), an initial angle of attack of $\gamma=3^{\circ}$ might be large enough to provoke a turbulent boundary layer along the whole trajectory of the arrow, resulting in a final deviation from the center of the target of around $\delta r=0.35 \mathrm{~m}(\mathbf{\square})$.

Due to the purely side-wind effect, the arrow shows only a lateral deviation or wind drift. For the cases of tail- $(-)$ and head-winds (-) with zero initial angular velocity, the final deviations from the center of the target are around $\delta r=0.10 \mathrm{~m}$ 
$(\triangleright)$ and $\delta r=0.11 \mathrm{~m}(\triangleleft)$, respectively, which implies that the side-wind affects in a more important way the trajectory of arrows in free-flight.

Consider the cases for which the ideal initial angular velocity is set in the computations, inserted panel in the inferior part of Figure 5, now the arrow almost aligns with the wind component and an angle attack close to zero is developed. The value of $\gamma$ remains small enough to keep a laminar boundary layer and the deviation from the center of the target reduces to around $\delta r=0.19 \mathrm{~m}(\bullet)$ for the side-wind. By keeping the arrow aligned with the wind flow and therefore the boundary layer laminar, it is possible to reduce the lateral deviation in the trajectory. Nevertheless, shooting the arrow with the ideal initial conditions might be challenging for the archers. Recently, Miyazaki et al. (2017) reported their experience in trying to adjust and control the arrows' initial angular velocity with little success (Miyazaki et al. 2017).

Figure 5. Main Area: Time Evolution of the Angle of Attack Considering Side-, Head-and Tail-Winds with a Velocity of $3 \mathrm{~ms}^{-1}$ for the Cases with Zero Initial Angular Velocity and Type-A Arrow. Inferior Panel: Cases with Ideal Initial Angular Velocity. Inserted Panel in the Right: Striking Points in the Target for All the Cases

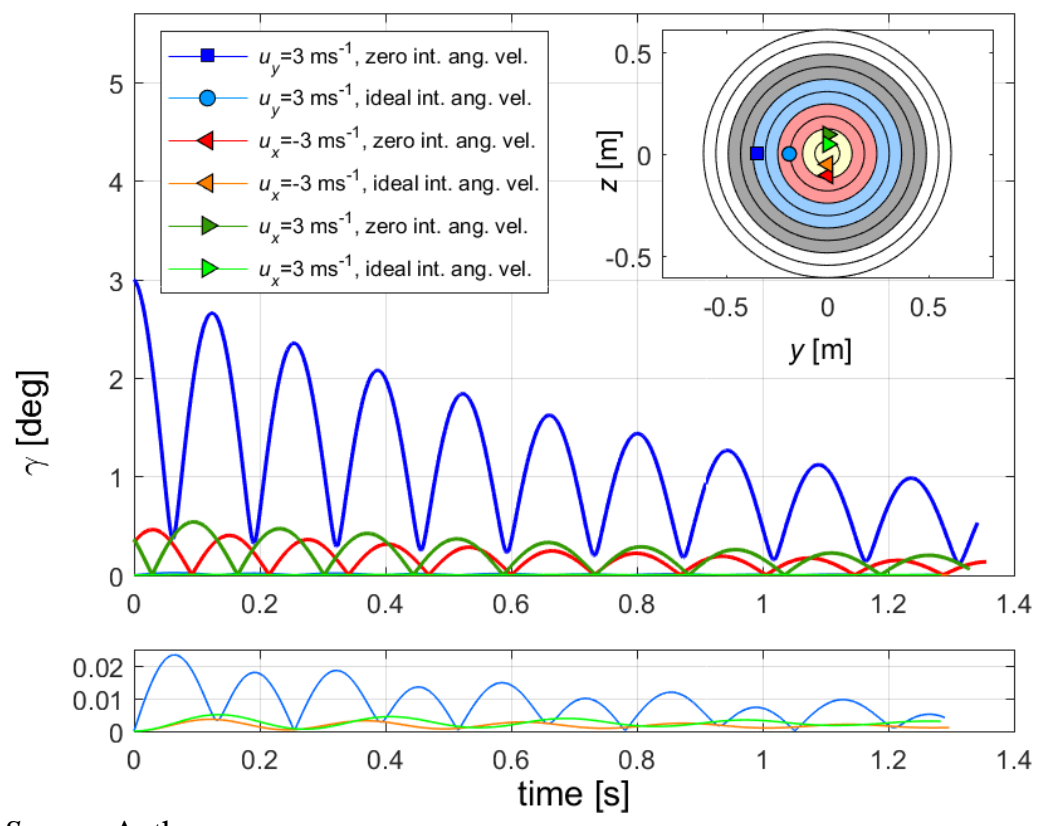

Source: Authors.

In Figure 6, it is shown that by increasing the arrow's initial velocity, $V_{0}$, it is possible to reduce the lateral deviation in the trajectory. Zero initial angular velocity and a uniform side-wind of $3 \mathrm{~ms}^{-1}$ was considered in the computations (and - ). If the typical velocity $\left(V_{0}=57 \mathrm{~ms}^{-1}\right)$ for arrows shot with recurve bows is increased $\sim 25 \%$, a reduction in the lateral deviation of $\sim 0.05 \mathrm{~m}$ and $\sim 0.07 \mathrm{~m}$ can be expected for the heavier arrow and the lighter one, respectively. Arrows shot with compound bows generally have larger initial velocities, $V_{0}>80 \mathrm{~ms}^{-1}$, resulting in less deviated trajectories than those shot with recurve bows (Park 2011). 
Considering that the lateral deviation in the trajectory or wind drift is mainly provoked by the lateral component of the drag, a rough estimation of the wind drift can be approximated by

$\delta y \sim \frac{F_{\mathrm{D}}}{2 M} \frac{u_{y}}{\sqrt{V_{0}^{2}+u_{y}^{2}}}\left(\frac{L}{V_{0}}\right)^{2} \sim \frac{C_{D} \rho \pi r^{2}}{4 M} \frac{u_{y}}{V_{0}} L^{2}$,

where $F_{\mathrm{D}}$ is the drag force and $L$ is the length of the archery range. In Figure 6 the results from the estimation ( - and - ) are plotted along with the results from the numerical computations for both types of arrows. The difference from the estimation and the numerical results arises from the assumption that in Equation (13) the velocity $V_{0}$ remains constant along the whole arrow's trajectory, whereas in an actual shot, the arrow's velocity was confirmed to reduce $\sim 15 \%$ and $\sim 12 \%$ for the type-A and type-B, respectively.

Figure 6. Dependence of the Lateral Deviation in the Trajectory on the Initial Arrow's Velocity. Not Ideal Initial Angular Velocity and a Side-wind of $3 \mathrm{~ms}^{-1}$ were considered

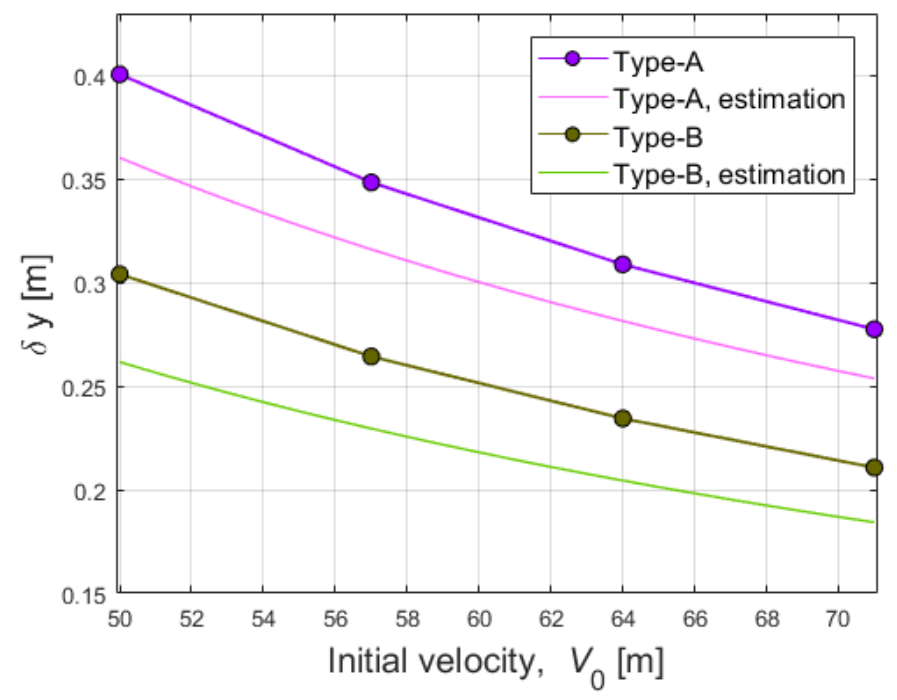

Source: Authors.

\section{Influence of the Mass in a Changing Wind Field}

Figure 7 shows an upper view of the computed trajectories for the type-A (Figure 7a) and type-B (Figure 7b) arrows with zero initial angular velocity. With multiple colours are represented the trajectories corresponding to the 6 different archery ranges. Multiple lines are plotted for different starting times with a delay of 1s between them. By changing the starting time of the shot, it is possible to make sure that the arrows are subject to different wind fields during free-flight. Regardless of the location and wind behaviour, the arrows with larger mass show less deviated trajectories (Figure 7b) than the lighter ones (Figure 7a). The lateral deviation in the trajectory decreases with increasing arrow's mass. 
The averaged $\delta y$ for each of the fields also change significantly, showing the different wind behaviour for each of the locations. In the final round area (fields I, II and III), positive side-winds are exerted on the arrows, resulting in positive values of $\delta y$. Whereas for the ranking round area (fields IV, V and VI) the negative deviations were found as a result of the negative side-winds $\left(u_{\mathrm{y}}<0\right)$. The average and standard deviation of $\delta y$ also varies from field to field. The averaged values of $\delta y$ resulted in $0.15 \mathrm{~m}, 0.11 \mathrm{~m}$ and $0.07 \mathrm{~m}$ for the fields I, II and III respectively for the lighter arrow (Figure 7a). Appreciably larger values of the standard deviation in $\delta y$ are present for the field V (-) compared with those of the field VI (-).

Figure 7. Computed Trajectories in All the Fields using Different Starting Times. Not Ideal Initial Angular Velocity was considered in All the Cases for (a) Type-A and (b) Type-B Arrows

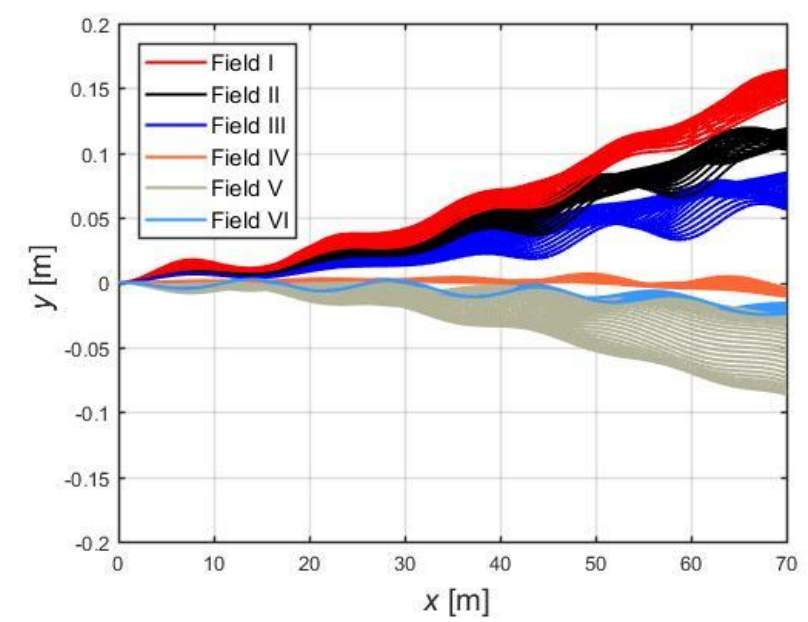

(a)

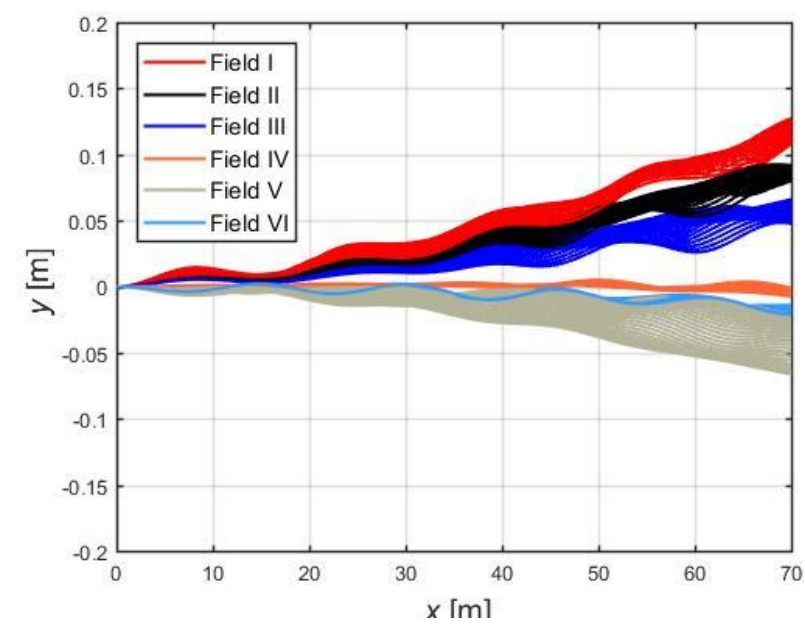

(b)

Source: Authors.

From the multiple shots with the type-A arrow and zero initial angular velocity were selected two cases in the final round area corresponding to the maximum and 
minimum $\delta y$, found in the fields I (-) and III (-), respectively. A difference in the lateral deviation of $\sim 0.10 \mathrm{~m}$ between such cases is not negligible and may affect in an important way the final score in the competition.

The wind velocity components, $u_{x}, u_{y}$ and $u_{z}$, experienced by the arrows in those two cases is plotted in Figure 8. As the arrows fly in the archery range, the wind strength changes with position. In the case of maximum $\delta y$ in the field I (Figure 8a), the maximum value of the side-wind $u_{y}$ is $2.07 \mathrm{~ms}^{-1}$. This side-wind velocity is exerted on the arrow for just a fraction of a second, in contrast to the cases of uniform side-wind, where the arrow experienced the same wind velocity along all the trajectory. For the case with minimum $\delta y$ in the field III (Figure $8 \mathrm{~b}$ ), the maximum side-wind velocity experienced by the arrow is $1.23 \mathrm{~ms}^{-1}$, from which the smaller $\delta y$ arises. The influence of the side-wind is emphasized because it appears to be more dangerous for the shots than the head- and tail-winds, as shown in the inserted panel in Figure 5.

Figure 8. Variation of the Wind Velocity with the Position Experienced by the Type-A Arrows showing Maximum and Minimum Values of dy in Fields I (a) and III (b)

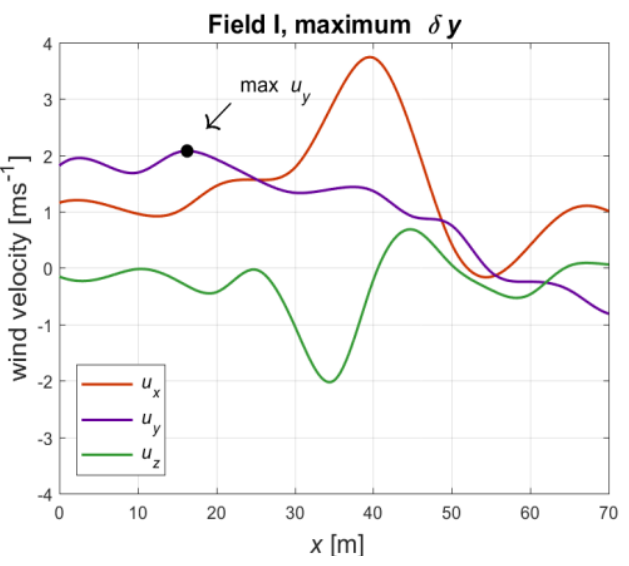

(a)

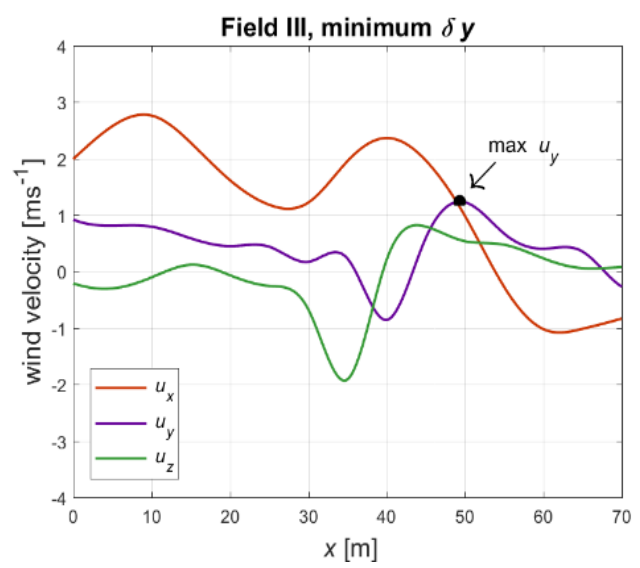

(b)

Source: Authors.

\section{Vertical Drop of Arrows Shot with the Ideal Initial Angular Velocity}

Figure 9 shows the time evolution of the angle of attack, $\gamma$, for a type-A arrow with the ideal initial angular velocities in the fields II and V with $\gamma_{\text {thr }}=0.4^{\circ}$. This implies that the boundary layer is initially laminar and becomes turbulent once $\gamma_{\text {thr }}$ is exceeded. There were performed computations for multiple starting times with a time difference of $1 \mathrm{~s}$ between them. The shots are shown with maximum ( - and a) and minimum ( - and $\bullet$ ) values of $\delta r$ for each field. It is indicated with $\bullet$ when the transition to turbulent boundary layer takes place. For the field II in Figure 9a with maximum $\delta r(\boldsymbol{\square})$, the transition to turbulent boundary occurs at $\sim 0.1 \mathrm{~s}$ from the shot, leading to a final radial deviation from the center of the target of $\delta r=0.31$ $\mathrm{m}$. For the case of minimum radial deviation $(\bullet), \delta r=0.14 \mathrm{~m}$, with a retarded transition to turbulent boundary layer, an improvement of more than $0.15 \mathrm{~m}$ could be achieved. 
Figure 9. Results for the Computations with Maximum and Minimum Values of $\delta r$ considering a Threshold Angle of Attack $\gamma_{\text {thr }}=0.4^{\circ}$ for the Fields I and V using a Type-A Arrow and Ideal Initial Angular Velocities (a) and (b) Show the Time Evolution of the Angle of Attack, $\gamma$. Inserted Panels: Impact Points on the Targets. Time Evolution of the Wind Components $\left(u_{x}, u_{y}\right.$ and $\left.u_{z}\right)$ Experienced by the Arrow along its Trajectory $(\boldsymbol{c}, \boldsymbol{d}, \boldsymbol{e}$ and $\boldsymbol{f})$

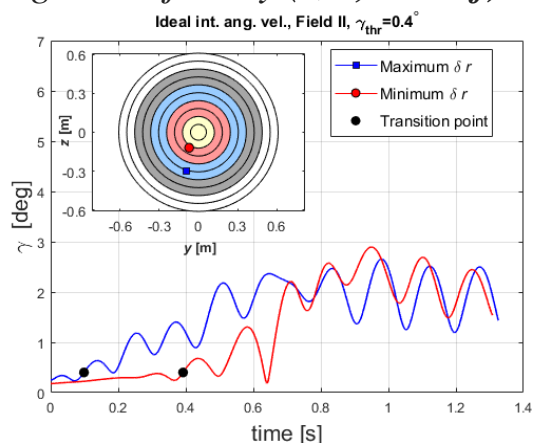

(a)

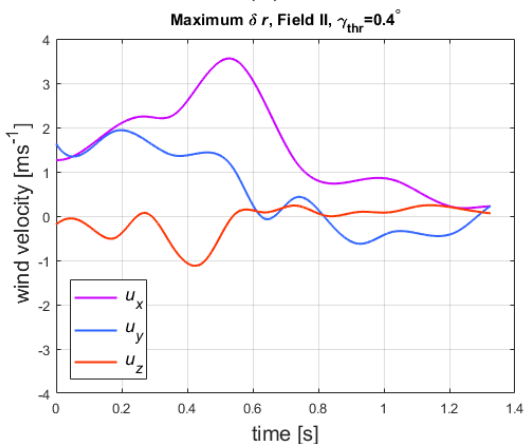

(c)

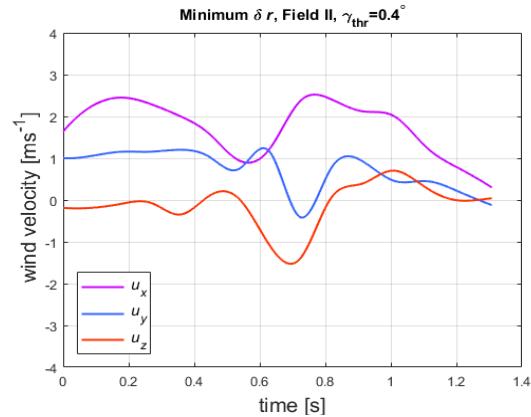

(e)

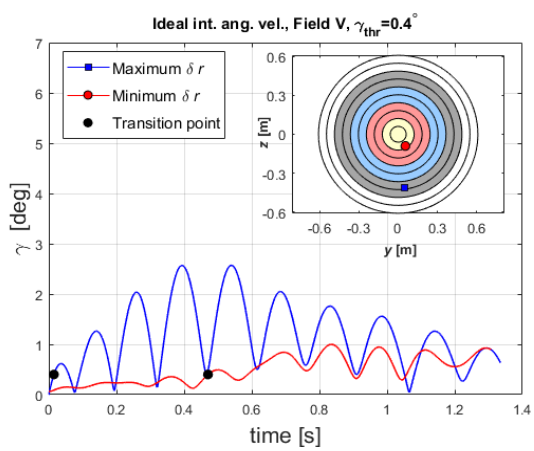

(b)

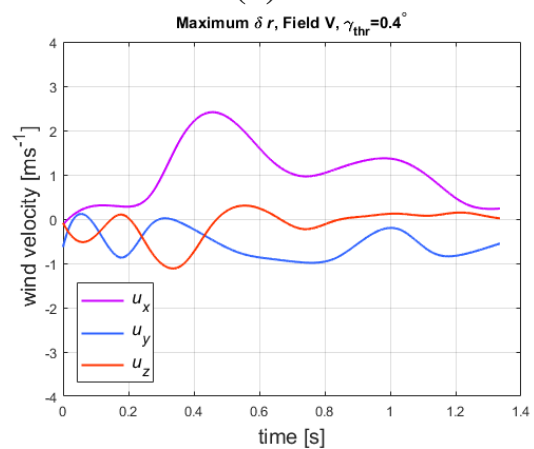

(d)

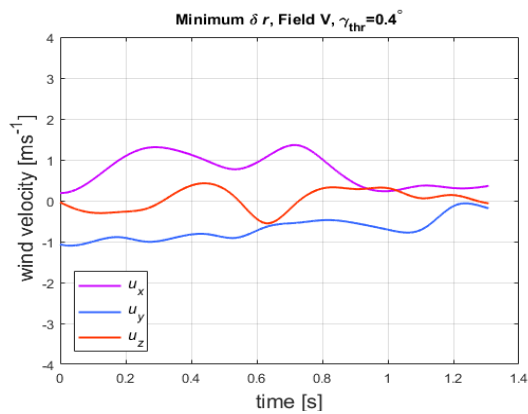

(f)

Source: Authors.

In Figure $9 \mathrm{~b}$ for the field $\mathrm{V}$, it is shown that delaying $\sim 0.44 \mathrm{~s}$ the transition to turbulent boundary layer may result in a significant reduction in the radial deviation from the center of the target. The obtained values are $\delta r=0.41 \mathrm{~m}$ and $\delta r=0.11 \mathrm{~m}$ for the earlier and the retarded transitions, respectively. Moreover, for the case with minimum $\delta r$, the value of the angle of attack remains small, $\gamma<1^{\circ}$, during all the flight ( - ), thus generating small drag and a less deviated trajectory.

In Figure 9c-f, the wind components experienced by the arrows with maximum and minimum values of $\delta r$ are shown. An earlier transition to turbulent boundary layer provokes that the arrows stay longer in the air, because of the 
larger drag force, thus contributing to the vertical drop in the trajectories. Further, in contrast to the cases with purely side-, head- and tail-winds, the non-zero vertical wind component $u_{\mathrm{z}}(-)$ also induces deviation in the $z$ direction.

\section{Discussion}

It has been proposed that the transition to turbulent boundary layer may take place when the maximum angle of attack is located in the range $0.4^{\circ}<\gamma_{\max }<0.6^{\circ}$ for $R e=1.75 \times 10^{4}$ (Miyazaki 2017). As $R e$ increases, the threshold angle at which the transition takes place decreases. Within the mentioned range, it is expected that exists a threshold angle, $\gamma_{\text {thr }}$, at which the transition occurs. In this section, the influence of the value of such $\gamma_{\text {thr }}$ is explored. Therefore, there were performed simulations considering transition to turbulent boundary layer with two different threshold values, $\gamma_{\text {thr }}=0.4^{\circ}$ and $\gamma_{\text {thr }}=0.6^{\circ}$, and compared with the case of a fully turbulent boundary layer.

Figure 10. Results for the Computations for a Type-A Arrow in the Field II using Two Different Threshold Values of the Angle of Attack $\left(\gamma_{t h r}=0.4^{\circ}\right.$ and $\gamma_{t h r}=0.6^{\circ}$ with Ideal Initial Angular Velocity) and Fully Turbulent Boundary Layer

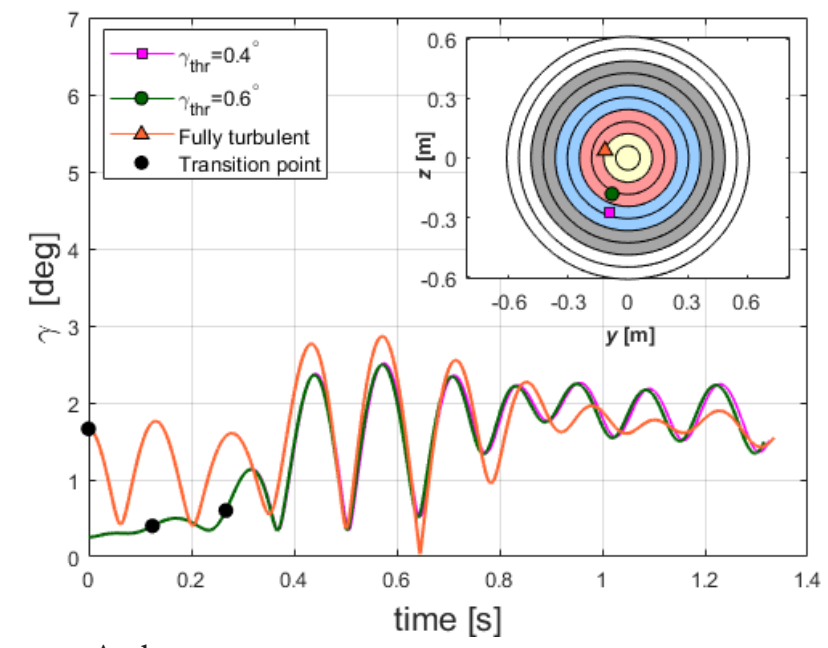

Source: Authors.

Figure 10 shows the time evolution of the angle of attack and in the inserted panel the target with the impact points on it. The ideal initial angular velocity was set for the cases with laminar-turbulent transition. On the other hand, a zero initial angular velocity was fixed in the fully turbulent computations. The characteristics of the type-A arrow were considered and the archery range where the shots were computed is the field II. For the case when $\gamma_{\text {thr }}=0.4^{\circ}$ was set ( - and $\square$ ), a turbulent boundary layer is developed at $\sim 0.12 \mathrm{~s}$ and results in a final deviation from the center of the target of $\delta r=0.29 \mathrm{~m}$.

On the other hand, when the threshold value was increased to $\gamma_{\text {thr }}=0.6^{\circ}$ (and $\bullet$ ), the transition to turbulent boundary layer $(\bullet)$ took place at $\sim 0.27 \mathrm{~s}$ after the shot was performed. For the latter case, the final deviation from the center of 
the target resulted in $\delta r=0.20 \mathrm{~m}$. Even though for both shots the boundary layer remains turbulent for most of the trajectory, a delay of $\sim 0.15 \mathrm{~s}$ in the transition resulted enough to reduce $\sim 0.09 \mathrm{~m}$ the radial deviation from the center of the target. Such reduction of the deviation in the trajectory might not be negligible to sum points in the final score. By keeping the boundary layer laminar the drag is reduced and therefore the deviation in the trajectory.

Consider now the case when the archer assumes zero initial angular velocity, i.e., not ideal initial conditions or turbulent boundary layer along the whole trajectory. In this case, the initial angle of attack presents a large enough value to generate a turbulent boundary layer from the beginning, $\gamma>1.6^{\circ}$, nevertheless the radial deviation from the center of the target is $\delta r=0.12 \mathrm{~m}$. Even larger drag force might be exerted due to the magnitude of $\gamma$, the final deviation resulted smaller than the cases with transition during the flight. The outcome appears to depend not only in the initial conditions, but also on the background wind characteristics. Even the archer was able to shoot the arrow with the ideal initial conditions, an unexpected wind behaviour along the flight resulted in large trajectory deviations.

\section{Conclusions}

In this paper, we investigated the influence of background winds on archery arrows using a mathematical model. The archery competitions are performed outdoors. Therefore, the background wind must be taken into because it represents one of the most important elements disturbing the shots.

We assumed the arrows to behave as rigid bodies. By computing their attitude, it was possible to obtain the time evolution of the angle of attack, from which the state of the boundary layer was inferred.

In our simulations, we introduced the background wind information that corresponds to the ground where the archery competitions will be held in the Olympic Games of Tokyo in 2020. Such wind information provided by JAMSTEC was the result of high-resolution large eddy simulations.

It was found that uniform side-wind disturbs more seriously the trajectory of arrows than the uniquely head- and tail-winds. Nevertheless, in an actual wind field the direction and wind velocity change with the location and time, resulting in unexpected arrows' behaviour.

Under a theoretical uniform side-wind, it appears feasible to keep the boundary layer laminar along the complete trajectory by aligning the arrows' attitude with the wind flow. By doing so, it is possible to reduce the wind drift.

The arrows' initial velocity and mass play a determinant role in the final deviation from the center of the target. With increasing initial velocity and mass, the arrows showed less deviated trajectories, regardless of the wind behaviour.

It was found that by delaying the transition from laminar to turbulent boundary layer, deviations in the trajectory were reduced under certain circumstances. The control of such transition has to be seriously considered. Furthermore, an unexpected behaviour of the wind during the arrows' flight may result in large deviations in their trajectory, even if the ideal initial conditions are achieved. Under 
non-uniform velocity wind fields, trying to keep the arrow's boundary layer laminar may be more harmful than beneficial.

\section{Acknowledgments}

The authors are deeply grateful to Dr. Onishi R and Dr. Matsuda K, from JAMSTEC, for their valuable support giving access to their high-resolution wind simulations. This work was partially supported by Grant in Aid for Scientific Research (C) 18k03943.

\section{References}

Allen T, Goff J (2018) Resources for sports engineering education. Sports Engineering 21(4): 245-253. DOI= https://doi.org/10.1007/s12283-017-0250-1.

Barton J, Vcelák J, Torres-Sanchez J, O'Flynn B, O'Mathuna C, Donahoe RV (2011) A miniaturised arrow ballistic measurement system. In 2018 IEEE Electron Devices Kolkata Conference (EDKCON), 1289-1292. Limerick, Ireland: IEEE. DOI=10.11 09/ICSENS.2011.6127412.

Barton J, Vcelák J, Torres-Sanchez J, O’Flynn B, O’Mathuna C, Donahoe RV (2012) Arrow-mounted ballistic system for measuring performance of arrows equipped with hunting broadheads. Procedia Engineering 34(Mar): 455-460. DOI=https://doi.org/ 10.1016/j.proeng.2012.04.078.

Goff, J (2013) A review of recent research into aerodynamics of sports projectiles. Sports Engineering 16(3): 137-154. DOI= https://doi.org/10.1007/s12283-013-0117-z.

Haake S (2009) The impact of technology on sporting performance in Olympic sports. Journal of Sports Science 27(13): 1421-1431. DOI=https://doi.org/10.1080/0264041 0903062019.

Hubbard M (1984) Optimal javelin trajectories. Journal of Biomechanics 17(10): 777-787. DOI= https://doi.org/10.1016/0021-9290(84)90108-8.

Hubbard M, Rust H (1984) Simulation of javelin flight using experimental aerodynamic data. Journal of Biomechanics 17(10): 769-776. DOI=https://doi.org/10.1016/00219290(84)90107-6,

Jung A, Muller W, Staat M (2018) Wind and fairness in ski jumping: a computer modelling analysis. Journal of Biomechanics 25(75): 147-153. DOI=10.1016/j.jbio mech.2018.05.001.

Kooi BW, Sparenberg J (1997) On the mechanics of the arrow: Archer's Paradox. Journal of Engineering Mathematics 31(2): 285-303. DOI=https://doi.org/10.1023/A:100426 2424363.

Kooi BW (1998) Bow-arrow interaction in archery. Journal of Sports Science 16(8): 721731. DOI= https://doi.org/10.1080/026404198366353.

Matsuda K, Onishi R, Takahashi K (2018) Tree-crown-resolving large-eddy simulation coupled with three-dimensional radiative transfer model. Journal of Wind Engineering and Industrial Aerodynamics 173(Feb): 53-66. DOI= https://doi.org/10.1016/j.jweia. 2017.11.015.

Miyazaki T, Mukaiyama K, Komori Y, Okawa K, Taguchi S, Sugiura H (2013). Aerodynamic properties of an archery arrow. Sports Eng. 16, 1, 43-54. DOI= https:// doi. org/10.1007/s12283-012-0102-y. 
Miyazaki T, Matsumoto T, Ando R, Ortiz J, Sugiura H (2017) Indeterminacy of drag exerted on an arrow in free flight: arrow attitude and laminar-turbulent transition. European Journal of Physics 38(6). DOI= https://doi.org/10.1088/1361-6404/aa8339.

Ortiz J, Ando M, Murayama K, Miyazaki T, Sugiura H (2019) Computation of the trajectory and attitude of arrows subject to background wind. Sports Engineering 22(7). DOI=http://doi.org/10.1007/s12283-019-0302-9.

Park J (2011) Minimizing wind drift of an arrow. Proceedings of the Institution of Mechanical Engineers Part P: Journal of Sports Engineering and Technology 226(1): 52-60. DOI= https://doi.org/10.1177/1754337111418876.

Park J, Hodge M, Al-Mulla S, Sherry M, Sheridan J (2011) Air flow around the point of an arrow. Proceedings of the Institution of Mechanical Engineers Part P: Journal of Sports Engineering and Technology 227(1): 64-69. DOI=https://doi.org/10.1177/17 54337111430569 .

Park J (2019) The impact of the atmosphere on target archery. Proceedings of the Institution of Mechanical Engineers Part P: Journal of Sports Engineering and Technology (Jan). DOI= https://doi.org/10.1177/1754337118823967.

Yaghoobian N, Mittal R (2018) A computational approach for predicting plant canopy induced wind effects on the trajectory of golf shots. Sports Engineering 21(1): 1-10. DOI= https://doi.org/10.1007/s12283-017-0239-9.

Zaniewski I (2009) Modeling of the archery bow and arrow vibrations. Shock and Vibration 16(3): 307-317. DOI= 10.3233/SAV-2009-0470. 